\title{
Face Recognition based on STWT and DTCWT using two dimensional Q-shift Filters
}

\author{
Sateesh Kumar H C*, C Chowda reddy**, Raja K B** and Venugopal K R** \\ *Department of Electronics and Communication Engineering, Sai Vidya Institute of Technology, Bangalore, \\ India \\ **University Visvesvaraya College of Engineering, Bangalore University, Bangalore, India
}

\begin{abstract}
The Biometrics is used to recognize a person effectively compared to traditional methods of identification. In this paper, we propose a Face recognition based on Single Tree Wavelet Transform (STWT) and Dual Tree Complex Wavelet transform (DTCWT). The Face Images are preprocessed to enhance quality of the image and resize. DTCWT and STWT are applied on face images to extract features. The Euclidian distance is used to compare features of database image with test face images to compute performance parameters. The performance of STWT is compared with DTCWT. It is observed that the DTCWT gives better results compared to STWT technique.

Index Terms: Bio metrics, DTCWT, STWT,ED, Two dimensional Q-shift filters, Face recognition
\end{abstract}

\section{INTRODUCTION}

The biometrics is used to recognize a person based on physiological and behavioral traits. The physiological traits are fingerprint, iris, face, palm print, DNA etc., are depends on physical characteristics of a person and are almost constant throughout lifetime. The behavioral traits are signature, keystroke, voice, gait etc., are depends on behavioral characteristics of a person and are not constant in the lifetime of a person and are depends upon mood and circumstances. The advantages of biometrics identification are biometric traits cannot be lost or forgotten or stolen as they are permanently attached to persons. The biometrics can be used as long as it satisfies universality, distinctiveness, permanence, collectability and acceptability.

The face biometric trait is powerful among all biometric traits as samples of face images are acquired using nonintrusive method and without any cooperation of a person. The steps adopted in face recognition are (i) acquire of face images, (ii) preprocessing,(iii) feature extraction, (iv) matching. In preprocessing the face color images are converted into gray scale images, image resize, illumination compensation, angle rotation etc., are performed. In feature extraction, the spatial domain features are extracted directly from an image, the transform domain features are extracted by converting spatial domain image into transform domain image using transforms such as Fast Fourier Transform (FFT), Discrete Cosine Transform (DCT), Short Time Fourier Transform (STFT), Discrete Wavelet Transform (DWT), DTCWT etc. In matching section the features of test images are compared with features of face images in the database using Euclidian
Distance (ED), Hamming Distance (HD), chi-square, Neural Network (NN), Support Vector Machine (SVM), Linear Discriminant Analysis (LDA), and Random Forest (RF) etc.

The biometrics is used in financial transactions, property documents, intellectual property protection, law enforcement, medical records, access to computer and internet etc.

Contribution: In this paper, face recognition based on STWT and DTCWT are compared. The features of face images are extracted using STWT and DTCWT. It is used to compute performance parameters.

Organization: This paper is organized into following sections. Section II is an overview of related work. The proposed model is described in Section III. Performance Analysis of the system is presented in Section IV and Conclusions are contained in Section $\mathrm{V}$ and references are given in section VI

\section{RELATED WORK}

Alaa Eleyan et al., [1] have introduced a face recognition method using DTCWT. PCA is used for face classification which is a linear dimensionality reduction technique. The DTCWT gives better result compares to Gabor wavelet transforms in terms of recognition rate. Zhongxi Sun et al., [2] have proposed a method for face recognition using DTCWT features with 2DPCA. DTCWT has advantage over DWT as it provides local multi-scale description of images with good directional selectivity and shift invariance. DTCWT is robust to illumination variations and facial expression changes. Results have shown that using this technique it's possible to capture the local information of different position, orientation and scales. Sun Yuehui and Du 
Minghui [3] described a method that combines two techniques DT-CWT and EMD for the illumination removal for face recognition. DT-CWT provides good selectivity in six directions. The presented technique is verified with PCA and LDA. Results shows that EMD is a better approach in normalizing a facial image in both space and frequency domain.

Jaya Priya and Rajesh [4] have explained a method in which the image is divided into several non-overlapped parallelogram blocks instead of square or rectangle. The complex wavelet coefficients, local mean and standard deviations are used for face recognition. Compared to Local DT CWT defined on squared structure, Local DT-CWT on parallelogram structure has more potential for perspective invariant face recognition. Ravi J et al.,[5] proposed the method in which the original face image is preprocessed and five level DT-CWT is applied in ordered to get DT-CWT coefficients. The $3 \times 3$ matrix is considered, for each of these matrix local binary patterns is used to get the final features and comparison of features of test image with database images are done using Euclidean Distance. The improvement in the result is shown in terms of False Rejection Rate (FRR), False Acceptance Rate (FAR) and Total Success Rate(TSR). Nick Kingsbury [6], has discussed a method to design a filters for DTCWT. The filters are designed such that both the filers are of even length and also time-reverse of each other. The filters have additional property that the group delay between the filter is one quarter of the sample period.

Zeenathunis a et al., [7] proposed a biometric identification system for frontal static face image subjected to various dark illu minations the frame work has preprocessing method, local feature extractor and a recognizer. An automatic Face Recognition Biometric System (FRBS) has been developed that uses Local Binary Pattern (LBP) and k - Nearest Neighbor classifier. The results for Yale $-\mathrm{B}$ database show that the use of LBP and $\mathrm{k}-\mathrm{NN}$ is able to improve the face recognition performance in various dark illuminations. Seyed Omid Shahdi and AbuBakar [8] proposed a method to recognize non-frontal faces with high performance by relying only on single full frontal gallery faces. By utilizing only small regions of the face or patches, the Fourier coefficients of these patches for each image are computed and transform them into a single vector. Instead of comparing and matching pixels values, the vectors are used to form a linear relationship which is then used to estimate the frontal face vector and then compare it with the actual frontal feature vector. Ngoc-Son $\mathrm{Vu}$ and Alice Caplier [9] describes Patterns of Oriented Edge Magnitudes (POEM) the recent feature descriptor, the feature descriptor is desired to be discriminative, robust and computationally inexpensive in both terms of time and storage requirement. They enhanced the performance of POEM with respect to all these criteria. Initially optimize the parameters of POEM and then apply the whitened principal-component-analysis dimensionality reduction technique to get a more compact, robust and discriminative descriptor. For face recognition, the efficiency of algorithm is strong for both constrained and unconstrained data sets in addition to low comple xity.

Harin sellahewa and sabah A Jassim [10] proposed a quality based adaptive approach to face recognition. This approach consists of three steps, firstly illumination quality of the given face image is used to decide if image should be preprocessed to normalize its illumination. Secondly, the global quality based normalization is extended to regional quality-based approach to adaptive illumination normalization. Thirdly, the illumination quality measure is used as a means to adaptively select the weighing parameters of the fused wavelet-based multistream face recognition. Future work is to investigate other as pects of face image quality such as facial expression, pose, and occlusion, such quality measures are used in fully adaptive face recognition system, which will select the most suitable gallery images, an appropriate face feature representation, and classification algorith $\mathrm{m}$ for given probe image and then able to predict the confidence of the system.

Ping-Han Lee et al., [11] proposed orientated local histogram equalization (OLHE) technique, that compensates illumination by encoding more information on the edge orientations and argued that edge orientation is useful for face recognition. Three OLHE feature combination methods are proposed for face recognition: one encoded most edge orientations; one was more compact with good edge-preserving capability, the performed well when extreme lighting conditions occur. They also showed that LBP is a special case of OLHE and OLHE is more effective than LBP for face recognition. The computational complexity of OLHE is less compared to state-of-theart algorith $m s$ such as logarith $m$ total variation model (LTV) that involves additional chain of preprocessing or total variation quotient image model (TVQI) that requires solving a variational problem.

Vishal M. Patel et al., [12] proposed an algorith $m$ to perform face recognition across varying illumination and pose based on learning small sized class specific dictionaries. This method consists of two main stages. In the first stage, given training samples from each class, class specific dictionaries are trained with some fixed number of atoms (elements of a dictionary). In the second stage, test face image is projected onto a span of the atoms in each learned dictionary. The residual vectors are then used for classification. Assuming the Lambertian reflectance model for the surface of a face, relighting approach is integrated within this framework so that 
many elements are added to gallery and robustness to illumination and pose changes can be realized. This method has the ability to recognize faces even when only a single or a few images are provided for training. Abhijith Punnappurath and Ambasamudram Narayanan Rajagopalan [13] proposed a face recognition technique under non uniform blur, Illumination and pose. The blurred images are modelled as a convex set. Illumination variations are handled by exploiting the fact of non uniform blurred and changed illumination image forms. The disadvantage of this technique is large changes in the face and significant occlusions cannot be handled. Changxing Ding et al.,[14] proposed robust face recognition technique to variation in the pose, expression and illumination. This method extracts multi directional, multi level dual-cross patterns. Difference in illumination is addressed by taking first derivative of Gaussian operator. It increases the robustness in pose variation and expression variation but it doubles the cost of local binary pattern.

\section{III.PROPOSED MODEL}

In this section, the face recognition is based on Single Tree Wavelet Transform (STWT) of Dual Tree Complex Wavelet transform (DTCWT) and DTCWT using two-dimensional Q-shift filters are discussed. The proposed Face recognition model is as shown in figure 1

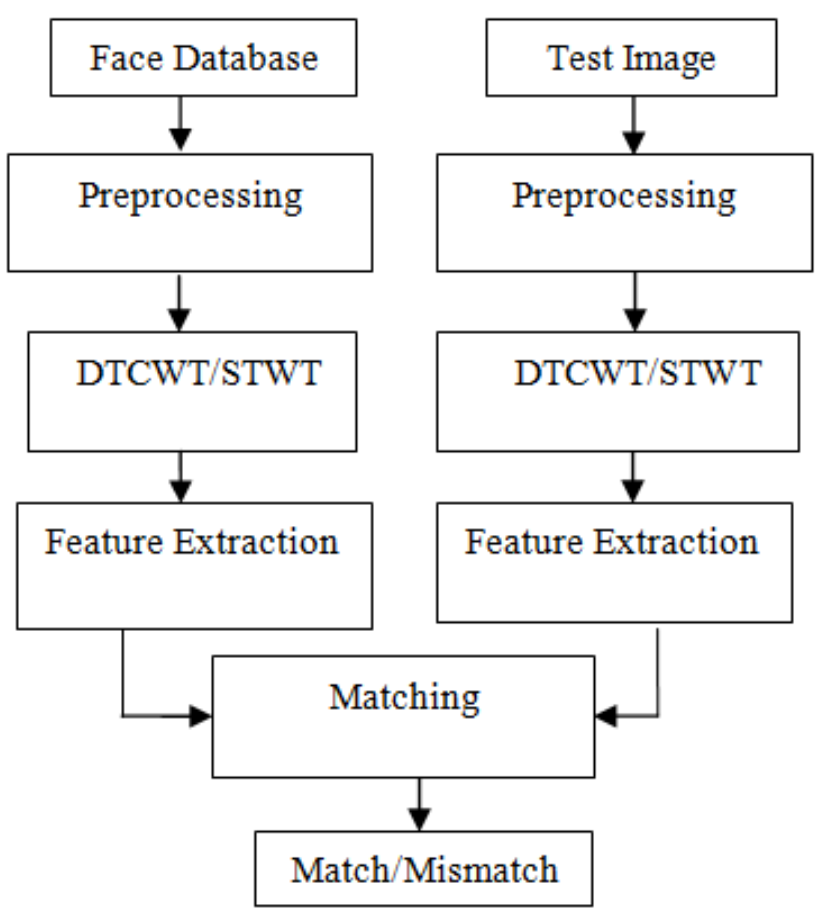

Fig.1: Face Recognition Model

3.1 Face Databases: The available face databases with various combinations of Persons inside Database (PID) and Persons Outside Database (POD) such as L-spacek, Yale, Near Infrared, ORL, JAFFE and Indian database are used to test the performance of proposed model.

3.1.1 L-Spacek Face database: The total number of persons in the database are hundred and nineteen with nineteen images per person. The database contains both male and female images with different face expressions. The size of each image sample is $320 * 280$ with bitmap format and face image samples are shown in figure 2 .

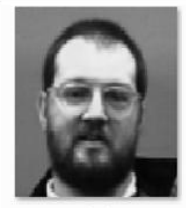

11

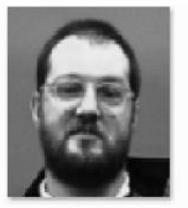

12

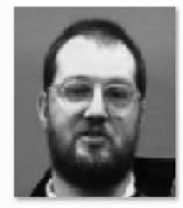

13

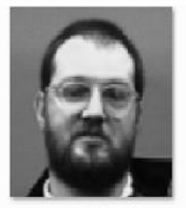

1.4
Fig.2: Samples of L- Spacek face images of a person

The first fifteen samples of every person are considered to create PID and seventeenth image of the every person from PID is considered as the test image to compute Total Success Rate (TSR) and False Rejection Rate (FRR).The Seventh image of every person from POD is considered as test image to compute False Acceptance Rate (FAR). 
3.1.2 Yale Face database: The face database include variations of pose, expression, Illu mination, scale and blurring. It has fifteen persons of male images with ten images per person totalling to one hundred and fifty images of size 320 x243 each with JPEG format. The samples of Yale face images of persons with different poses is shown in figure 3 . The first 8 face images of every person are considered to creating PID. The tenth image of every person from PID is considered as the test image to compute TSR and FRR. The tenth image of every person from POD is considered to compute FAR.
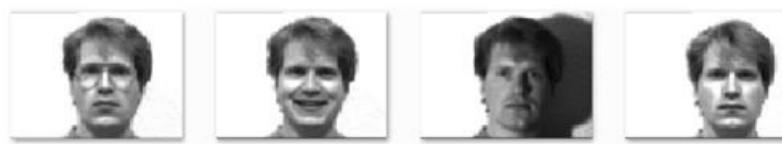

Fig.3: Samples of Yale face images of person

3.1.3 Near Infrared (NIR): The database includes variations of pose, expression, illumination, scale, blurring and a combination of them. The database consists of 115 persons with 15 images per person. This standard database contains both male and female images with and without spectacles. The face images are of JPEG format with each image of size 768*576. The samples of NIR face images are shown in figure 4. The first twelve face images of every person are considered in creating PID. The thirteenth image of the every person from PID is considered as the test image to compute TSR and FRR. The thirteenth image of every person from POD is considered as tes $t$ image to co mpute FAR.
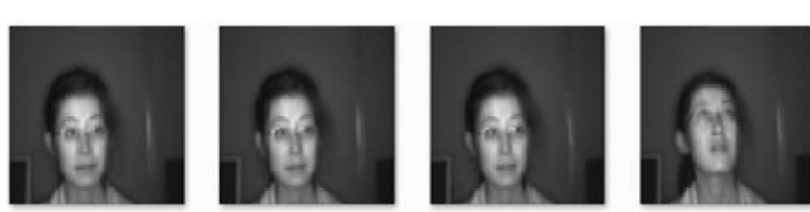

Fig.4: Samples of NIR face images of a person

3.1.4 Olivetti Research Lab (ORL) database: The face database consists of forty persons with ten images per person, with no color images. All images are of PGM format with each image of size $92 * 112$. The images are captured with slight tilt of the head. The samples of ORL face images are shown in figure 5. The first nine face images of every person are considered in creating PID. The tenth image of the every person from PID is considered as the test image to compute TSR and FRR. The tenth image of every person from POD is considered as test image to compute FAR.
3.1.5 Japanese Female Facial Expression (J AFFE): The face database consists of ten persons with approximately twenty images per person. All images are of TIFF format with each image of size $256 * 256$.The samples of JAFFE face database is shown in figure 6.
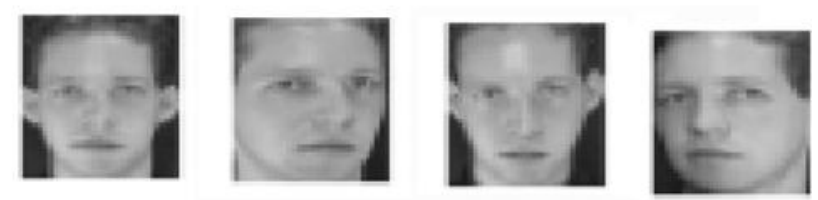

Fig.5: Samples of ORL face images of a person.

The first seventeen face images of every person are considered in creating PID. The nineteenth image of the every person from PID is considered as the test image to compute TSR and FRR. The nineteenth image of every person from POD is considered as test image to compare FAR.
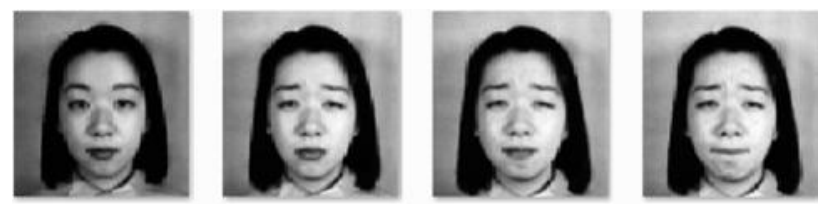

Fig.6: Samples of JAFFE face images of a person

3.1.6 Indian male's database: The face database consists of twenty persons with eleven images per person. This database contains color images with smile, left shift, right shift and more orientations. All images are of JPEG format with each image of size $640 * 480$. The first eight face images of every person are considered in creating PID. The $n$ inth image of the every person from PID is considered as the test image to compute TSR and FRR. The ninth image of every person from POD is considered as test image to compute FAR. The samples of Indian males face database is shown in figure 7
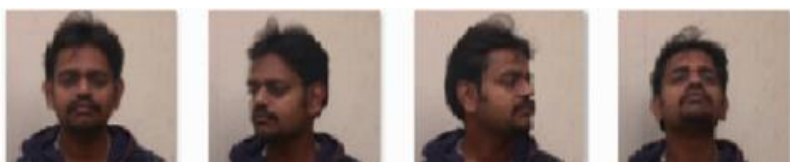

Fig.7: Samples of Indian males face images of a person

3.1.7 Indian female database: The face database consists of twenty two persons with eleven images per person. This database contains color images with smile, left shift, right shift and more orientations. All images are of JPEG format with each image of size $640 * 480$. 


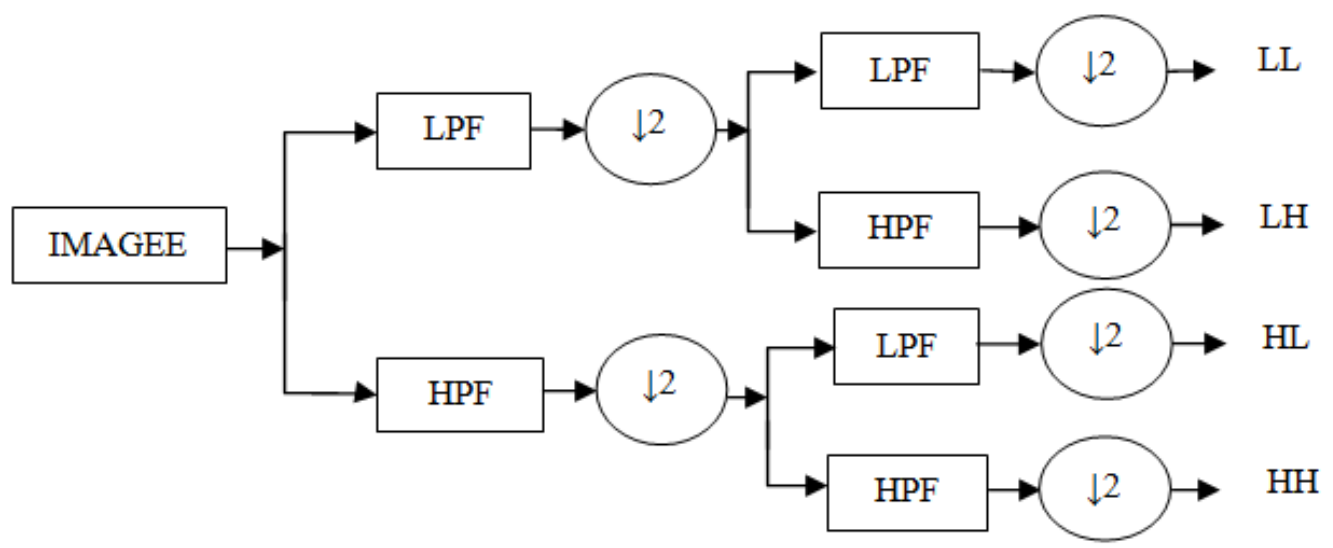

Fig. 9 Single Tree Wavelet Transformation

The first eight face images of every person are considered in creating PID. The eleventh image of the every person from PID is considered as the test image to compute TSR and FRR. The eleventh image of every person from POD is considered as test image to compute FAR. The samples of Indian females face database is shown in figure 8 .
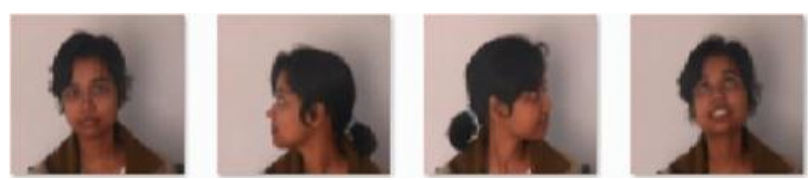

Fig.8: Samples of Indian females face images of a person

3.2 PRE-PROCESSING: The face image samples are preprocessed to convert color into gray scale face images and each image is resized to $256^{*} 256$.

3.3 STWT of DTCWT:The STWT is one tree of DTCWT, which is similar to DWT. Hence, DWT and STWT are used interchangeably wherever necessary. Wavelet transform is a powerful mathematical tool used to extract localized time-frequency information of an image.

The decomposition of the data into different frequency range is made using mother wavelet and scaling function and is reversible in its operation. The band pass filters perform the task of separation of frequency components

In one dimensional DWT, an image signal can be analyzed by passing through an analysis filter bank followed by decimation operation. The analys is filter bank consists of a low-pass and high-pass filter at each decomposition stage as shown in the fig. 9 . When signal passes through these filters, it splits into two bands. The low-pass filter, which corresponds to an averaging operation, extracts the approximate information of the signal. The high pass filter, which corresponds to a differencing operation, extracts the detailed information of the signal. The output of the filtering operation is then decimated by two. A twodimensional transformation is obtained by performing two separate one-dimensional transforms. First, the image is filtered along the row and decimated by two. It is then followed by filtering the sub-image along column and decimated by two.

This operation splits the image into four bands, namely, LL, LH, HL and HH corresponding to approximation band, Horizontal band, vertical band and diagonal bands respectively. The DWT is an efficient computational algorithm but having four fundamental disadvantages such as Oscillations, Shift Variance, Aliasing and Lack of Directionality.

3.4 Dual Tree Complex Wavelet Transform (DTCWT): It is an enhancement technique to DWT with s mall additional properties and changes. It is an effective method for implementing an analytical wavelet transform, introduced by Kingsbury [6], [15]. Generating complex coefficients by DTCWT introduces limited redundancy and allows the transform to provide shift invariance and directional selectivity of filters.

The DTCWT employs two real DWTs; the first DWT is the real part of the complex transform while the second DWT is the imaginary part of the complex transform. The two wavelet transforms use two different sets of filters, with each satisfying the perfect reconstruction conditions The $h_{0}(n)$ and $\mathrm{h}_{1}(\mathrm{n})$ denote the low-pass and high-pass filter pair for the Upper Filter-Bank (UFB) of first set of wavelet transform. The $\mathrm{g}_{0}(\mathrm{n}) \operatorname{andg}_{1}(\mathrm{n})$ denote the low-pass and high-pass filter pair for the Lower Filter Bank (LFB) second set of wavelet transform. The two wavelets associated with each set of wavelet transforms are denoted as $\mathrm{w}_{\mathrm{h}}(\mathrm{t})$ and $\mathrm{w}_{\mathrm{g}}(\mathrm{t})$ The filters are designed to satisfy the perfect reconstruction for complex wavelet $\mathrm{w}(\mathrm{t})=\mathrm{W}_{\mathrm{h}}(\mathrm{t})+\mathrm{jw}_{\mathrm{g}}(\mathrm{t})$. The wavelet $\mathrm{w}_{\mathrm{g}}(\mathrm{t})$ is the Hilbert Transform of $\mathrm{W}_{\mathrm{h}}(\mathrm{t})$ and is the complex wavelet transform ie., $\mathrm{w}_{\mathrm{g}}(\mathrm{t}) \approx \mathrm{H}\left\{\mathrm{w}_{\mathrm{h}}(\mathrm{t})\right\}$ 


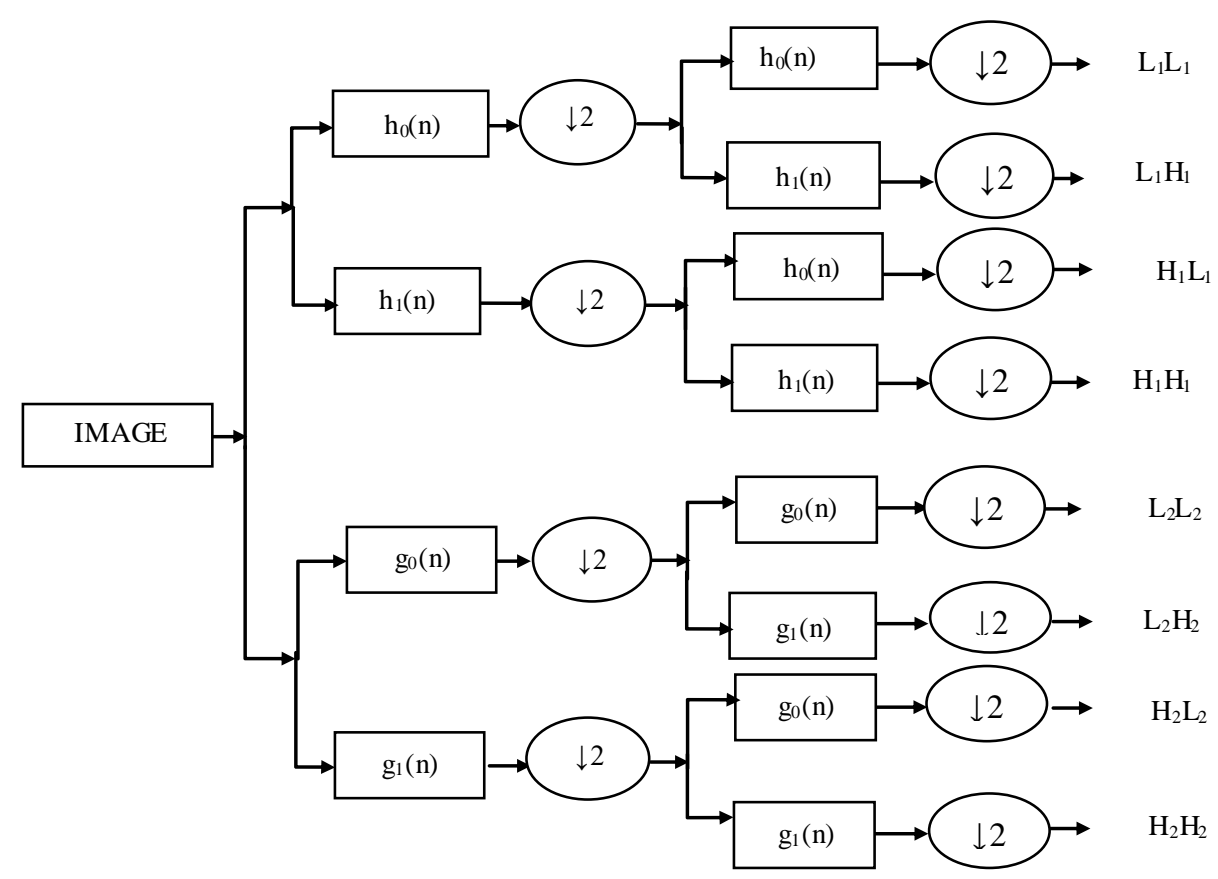

Fig.10 Dual Tree Complex Wavelet Transformation

When the DTCWT is applied to a real signal, the output of the UFB and LFB's are real and imaginary parts of the complex coefficients and stored separately. However, if the DTCWT is applied to a complex signal, then the outputs of both UFB and LFB's will be complex and no longer correct to label them as the real and imaginary parts. For a real $N$ point signal $2 N$ complex coefficients are obtained, but $N$ of these coefficients are the complex conjugates of the other $N$ coefficients. Q-shift method is used to design filters satisfying perfect reconstruction and approximately linear-phase condition with required group delay. The filter bank structure of 2D -DTCWT is shown in figure 10 .

The 2D structure requires four trees for analysis filter bank. The filter bank structure of tree-a is similar to 2D DWT. All other trees (b, c, and d) have similar structures with the appropriate combinations of filters, the overall 2D-DTCWT structure is 4-times expensive than standard 2D
DWT. The tree-a and ree-b form a real pair, while the tree-c and tree-d form the imaginary pair of the analysis filter bank. Each level of DTCWT provides 16 sub-bands with 4 sub-bands of low frequencies and 12 sub-bands of high frequencies as shown in the figure 11 .

3.5 Euclidian distance:The matching metric that can be

used to compare two feature templates of face images is the Euclidean distance. The Euclidian distance is given by an Equation (1)

$\mathrm{d} 1(\mathrm{p}, \mathrm{q})=\sqrt{\sum_{\mathrm{i}=1}^{\mathrm{M}}\left(p_{i}-q_{i}\right)^{2}}$

1)

Where, $M=$ The dimension of feature vector

The $i^{\text {th }}$ feature value of first face image

The $i^{\text {th }}$ feature value of second face image

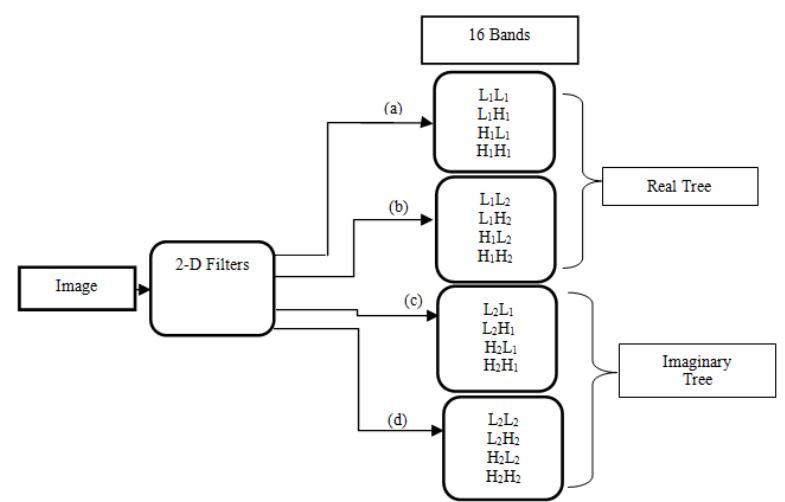

Fig. 11: Filter bank structure for 2D-DTCWT 


\section{ALGORITHM}

Problem definition: The face recognition performance parameters are analyzed using STWT and DTCWT by using two dimensional Q-shift filters.

The proposed algorithm is used (i) To increase the recognition rate (TSR).

(ii) To decrease FRR, FAR and EER.

(iii) To compare performance of two transforms.

The performance of the proposed algorithm is tested using STWT and DTCWT and is given in table 1

Table 1: The Proposed Algorithm

INPUT : Face images database, Test Face images

OUTPUT : Performance parameters

1. The different face databases are considered.

2. The face images are preprocessed.

3. The two dimensional filters are useded for STWT and DTCWT.

4. The STWT and DTCWT is applied on face image database to generate features.

5. The test face image is considered and features are generated using STWT and

DTCWT.

6. The features of test and database images are compared using ED.

7. The performance parameters are compared using STWT and DTCWT.

\section{RESULTS}

5.1 Euclidian distance comparison STWT and DTCWT for different Face databases: In this section, the ED between face images of same and different persons using STWT and DTCWT, performance evaluation of Face recognition using DTCWT, the variations of EER and optimum TSR for different combinations of PID and PODs and performance comparis on of STWT and DTCWT are discussed.

\subsubsection{ED Comparison using Approximation band:} The ED between two images of same person and two images of different persons using approximation bands of STWT and DTCWT with different face database images are given in Table 2 and Table 3 respectively. It is observed that the $\mathrm{ED}$ difference value between same and different persons are more in the case of DTCWT compared to STWT. The higher ED difference values indicate, better in differentiating same and different person images.

Table 2: ED difference values between persons using STWT for appro ximation Band (LL Band)

\begin{tabular}{|c|r|r|c|}
\hline Database & $\begin{array}{c}\text { Same } \\
\text { person }\end{array}$ & $\begin{array}{c}\text { Different } \\
\text { person }\end{array}$ & Difference \\
\hline L-specek & 12365 & 40465 & $\mathbf{2 8 1 0 0}$ \\
\hline Yale & 12129 & 45899 & $\mathbf{3 3 7 7 0}$ \\
\hline NIR & 14275 & 24866 & $\mathbf{1 0 5 9 1}$ \\
\hline ORL & 23069 & 41175 & $\mathbf{1 8 1 0 6}$ \\
\hline JAFFE & 20811 & 33693 & $\mathbf{1 2 8 8 2}$ \\
\hline $\begin{array}{c}\text { Indian } \\
\text { Male }\end{array}$ & 21351 & 22947 & $\mathbf{1 5 9 6}$ \\
\hline $\begin{array}{c}\text { Indian } \\
\text { Female }\end{array}$ & 13441 & 18474 & $\mathbf{5 0 3 3}$ \\
\hline
\end{tabular}

Table 3: ED difference values between persons using DTCWT for approximation Band (LL Band)

\begin{tabular}{|c|c|c|c|}
\hline Database & $\begin{array}{c}\text { Same } \\
\text { person }\end{array}$ & $\begin{array}{c}\text { Different } \\
\text { person }\end{array}$ & Difference \\
\hline L-speck & 634.378 & 1610.7 & 976.32 \\
\hline Yale & 1483.7 & 3129.1 & 1645.4 \\
\hline NIR & 1112 & 1314 & 202 \\
\hline ORL & 1329 & 1412 & 83 \\
\hline JAFFE & 2834 & 3466 & 632 \\
\hline Indian Male & 1314 & 2636 & 1322 \\
\hline $\begin{array}{c}\text { Indian } \\
\text { Female }\end{array}$ & 1258 & 1805 & 547 \\
\hline
\end{tabular}

5.1.2 ED Comparison using Detailed Bands: The features of LH, HL and $\mathrm{HH}$ bands are concatenated to generate final detailed band features. The ED between two images of same person and different persons using detailed bands of STWT and DTCWT with different face database images are given in Table 4 and Table 5 respectively. It is observed that the ED difference value between same and different persons are more in the case of DTCWT compared to STWT.It is observed from sections 5.1.1 and 5.1.2 that the ED difference values with approximation and detailed bands using DTCWT are higher compared to STWT which indicates, DTCWT is better in differentiating same and different person images. It is also observed that the ED difference with approximate band is high compare to detailed bands. Hence DTCWT with approximation band is better in face identification. 
Table 4: ED difference values between persons using STWT for Detailed Band (LH, HL and HH Bands)

\begin{tabular}{|c|c|c|c|}
\hline Database & Same person & Different person & Difference \\
\hline L-speck & 6339 & 20305 & 13966 \\
\hline Yale & 6244 & 23047 & 16803 \\
\hline NIR & 7201 & 12461 & 5260 \\
\hline ORL & 11635 & 20691 & 9056 \\
\hline JAFFE & 10624 & 17086 & 6462 \\
\hline $\begin{array}{c}\text { Indian } \\
\text { Male }\end{array}$ & 10760 & 11594 & 834 \\
\hline $\begin{array}{c}\text { Indian } \\
\text { Female }\end{array}$ & 6810 & 9323 & 2513 \\
\hline
\end{tabular}

Table 5: ED difference values between persons using DTCWT for Detailed Band (LH, HL and HH Bands)

\begin{tabular}{|c|c|c|c|}
\hline Database & Same person & Different person & Difference \\
\hline L-speck & 550.608 & 723.055 & 172.447 \\
\hline Yale & 1246.7 & 1829.6 & 582.92 \\
\hline NIR & 639.18 & 693.23 & 54.053 \\
\hline ORL & 541.098 & 544.868 & 3.77 \\
\hline JAFFE & 2182 & 2398 & 216 \\
\hline Indian Male & 823.91 & 1412.4 & 588.49 \\
\hline $\begin{array}{c}\text { Indian } \\
\text { Female }\end{array}$ & 757.35 & 1027.6 & 270.25 \\
\hline
\end{tabular}

5.2 Performance analysis using DTCWT for different face databases: The performance parameters such as FRR, FAR, TSR and EER are computed and analysed for different face databases in this section

5.2.1 Analysis using L-S pacek face database: The variations of FRR, FAR, and TSR with threshold for PID of sixty and POD of fifty nine for L-spacek face database is given in table 6 . The values of FAR and TSR increases as threshold values increases. The FRR values decreases with increase in threshold values. The EER value of $1.11 \%$ at 0.0053 threshold and the corresponding percentage TSR is 100 as shown in figure 12 .

5.2.2 Analysis using Yale face database: The variations of FRR, FAR, and TSR with threshold for PID of eight and POD of seven for Yale face database are given in table 7. The values of FAR and TSR increases as threshold values increases. The FRR value decreases with increase in threshold value. The maximum percentage TSR value is 87.5 and minimum values of FRR and FAR are zeros. The EER value is $6.667 \%$ at threshold value of 0.0104 and the corresponding percentage TSR is 87.5 as shown in figure 13

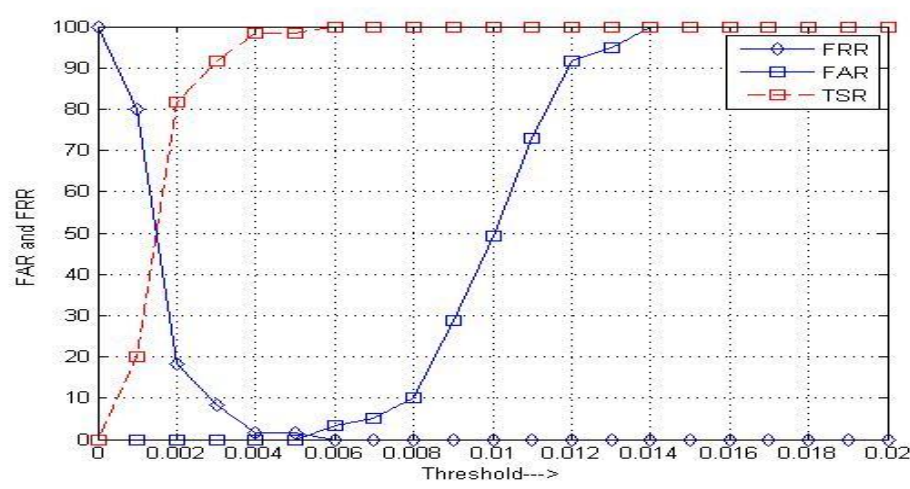

Fig. 12: Plot of FRR FAR and TSR with threshold values for PID:POD of 60:59 for L-spacek

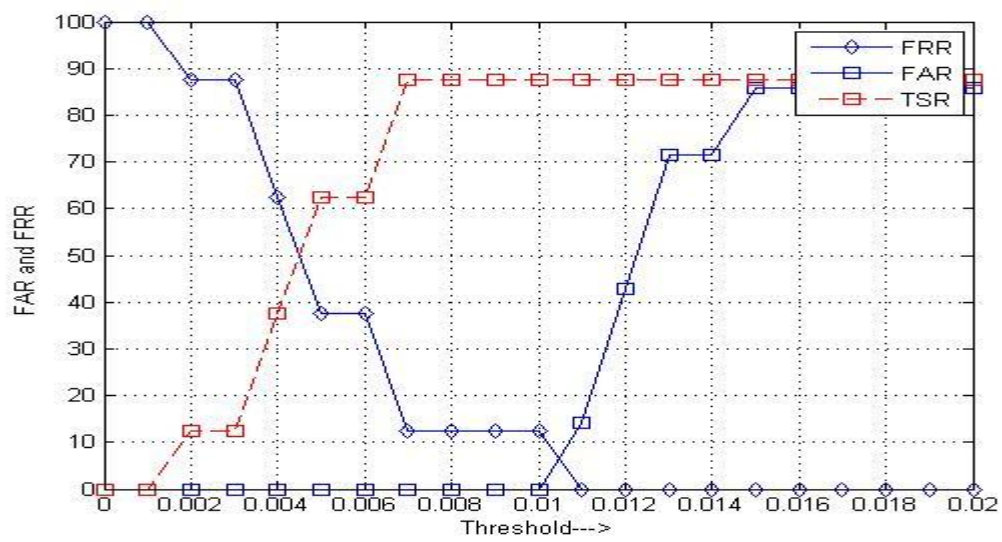

Fig.13: Plot of FRR FAR and TSR with threshold values for PID:POD of 8:7 for Yale database 
Table 6: Variations of FRR, FAR, TSR with threshold for PID:POD of 60:59 for L-Spacek

\begin{tabular}{|c|c|c|c|}
\hline Threshold & \%FRR & \%FAR & \%TSR \\
\hline 0 & 100 & 0 & 0 \\
\hline 0.001 & 80 & 0 & 20 \\
\hline 0.002 & 18.3333 & 0 & 81.6667 \\
\hline 0.003 & 8.3333 & 0 & 91.6667 \\
\hline 0.004 & 1.6667 & 0 & 98.3333 \\
\hline 0.005 & 1.6667 & 0 & 98.3333 \\
\hline 0.006 & 0 & 3.3898305 & 100 \\
\hline 0.007 & 0 & 5.0847458 & 100 \\
\hline 0.008 & 0 & 10.1694915 & 100 \\
\hline 0.009 & 0 & 28.8135593 & 100 \\
\hline 0.01 & 0 & 49.1525424 & 100 \\
\hline 0.011 & 0 & 72.8813559 & 100 \\
\hline 0.012 & 0 & 91.5254237 & 100 \\
\hline 0.013 & 0 & 94.9152542 & 100 \\
\hline 0.014 & 0 & 100 & 100 \\
\hline 0.015 & 0 & 100 & 100 \\
\hline 0.016 & 0 & 100 & 100 \\
\hline 0.017 & 0 & 100 & 100 \\
\hline 0.018 & 0 & 100 & 100 \\
\hline 0.019 & 0 & 100 & 100 \\
\hline 0.02 & 0 & 100 & 100 \\
\hline
\end{tabular}

Table 7: Variations of FRR, FAR, TSR with threshold for PID:POD of 8:7 for Yale database

\begin{tabular}{|c|c|c|c|}
\hline Threshold & \%FRR & \%FAR & \%TSR \\
\hline 0 & 100 & 0 & 0 \\
\hline 0.001 & 100 & 0 & 0 \\
\hline 0.002 & 87.5 & 0 & 12.5 \\
\hline 0.003 & 87.5 & 0 & 12.5 \\
\hline 0.004 & 62.5 & 0 & 37.5 \\
\hline 0.005 & 37.5 & 0 & 62.5 \\
\hline 0.006 & 37.5 & 0 & 62.5 \\
\hline 0.007 & 12.5 & 0 & 87.5 \\
\hline 0.008 & 12.5 & 0 & 87.5 \\
\hline 0.009 & 12.5 & 0 & 87.5 \\
\hline 0.01 & 12.5 & 0 & 87.5 \\
\hline 0.011 & 0 & 14.2857143 & 87.5 \\
\hline 0.012 & 0 & 42.8571429 & 87.5 \\
\hline 0.013 & 0 & 71.4285714 & 87.5 \\
\hline 0.014 & 0 & 71.4285714 & 87.5 \\
\hline 0.015 & 0 & 85.7142857 & 87.5 \\
\hline 0.016 & 0 & 85.7142857 & 87.5 \\
\hline 0.017 & 0 & 85.7142857 & 87.5 \\
\hline 0.018 & 0 & 85.7142857 & 87.5 \\
\hline 0.019 & 0 & 85.7142857 & 87.5 \\
\hline 0.02 & 0 & 85.7142857 & 87.5 \\
\hline
\end{tabular}

5.2.3 Analysis using NIR face database:The variations of FRR, FAR, and TSR with threshold for PID of sixty and POD of fifty five for NIR face database are given in table 8 .

Table 8: Variations of FRR, FAR, TSR with threshold for PID: POD of 60:55 for NIR

\begin{tabular}{|c|c|c|c|}
\hline Threshold & \%FRR & \%FAR & \%TSR \\
\hline 0 & 100 & 0 & 0 \\
\hline 0.001 & 43.3333333 & 0 & 56.66666667 \\
\hline 0.002 & 10 & 5.4545455 & 90 \\
\hline 0.003 & 6.6666667 & 32.7272727 & 91.66666667 \\
\hline 0.004 & 1.6666667 & 54.5454545 & 93.33333333 \\
\hline 0.005 & 1.6666667 & 63.6363636 & 93.33333333 \\
\hline 0.006 & 1.6666667 & 67.2727273 & 93.33333333 \\
\hline 0.007 & 1.6666667 & 74.5454545 & 93.33333333 \\
\hline 0.008 & 0 & 81.8181818 & 93.33333333 \\
\hline 0.009 & 0 & 89.0909091 & 93.33333333 \\
\hline 0.01 & 0 & 90.9090909 & 93.33333333 \\
\hline 0.011 & 0 & 94.5454545 & 93.33333333 \\
\hline 0.012 & 0 & 96.3636364 & 93.33333333 \\
\hline 0.013 & 0 & 96.3636364 & 93.33333333 \\
\hline 0.014 & 0 & 96.3636364 & 93.33333333 \\
\hline 0.015 & 0 & 96.3636364 & 93.33333333 \\
\hline 0.016 & 0 & 96.3636364 & 93.33333333 \\
\hline 0.017 & 0 & 98.1818182 & 93.33333333 \\
\hline 0.018 & 0 & 98.1818182 & 93.33333333 \\
\hline 0.019 & 0 & 98.1818182 & 93.33333333 \\
\hline 0.02 & 0 & 98.1818182 & 93.33333333 \\
\hline
\end{tabular}

The values of FAR and TSR increases as threshold values increases. 
The FRR values decreases with increase in threshold values. The maximum percentage TSR value is ninety three and minimu $m$ values of FRR and FAR are zeros. The EER value is $9.5 \%$ and corresponding TSR Value is $93.33 \%$ and plotted in figure 14 .

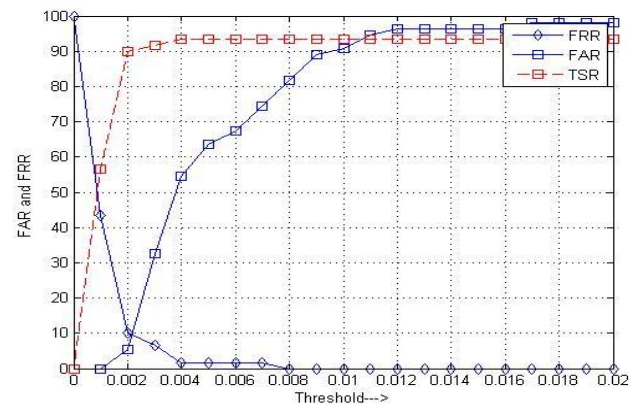

Fig.14: Plot of FRR FAR and TSR with threshold values for PID:POD of 60:55 for

NIR database

5.2.4 Analysis using ORL face database: The variations of FRR, FAR, and TSR with threshold for PID of twenty and POD of twenty for ORL face database are given in table 9. The values of FAR and TSR increases as threshold values increases. The FRR values decreases with increase in threshold values. The maximum percentage TSR value is 90 and minimum values of FRR and FAR are zeros. The EER value is $11.11 \%$ and the Corresponding TSR value is $85 \%$ as shown in figure 15 .

Table 9: Variations of FRR, FAR, TSR with threshold for PID: POD of 20:20 for ORL

\begin{tabular}{|c|c|c|c|}
\hline Threshold & \%FRR & \%FAR & \%TSR \\
\hline 0 & 100 & 0 & 0 \\
\hline 0.001 & 100 & 0 & 0 \\
\hline 0.002 & 95 & 0 & 5 \\
\hline 0.003 & 85 & 0 & 15 \\
\hline 0.004 & 65 & 0 & 35 \\
\hline 0.005 & 35 & 0 & 65 \\
\hline 0.006 & 15 & 10. & 85 \\
\hline 0.007 & 10 & 20 & 85 \\
\hline 0.008 & 0 & 40 & 90 \\
\hline 0.009 & 0 & 60 & 90 \\
\hline 0.01 & 0 & 70 & 90 \\
\hline 0.011 & 0 & 80 & 90 \\
\hline 0.012 & 0 & 90 & 90 \\
\hline 0.013 & 0 & 95 & 90 \\
\hline 0.014 & 0 & 95 & 90 \\
\hline 0.015 & 0 & 100 & 90 \\
\hline 0.016 & 0 & 100 & 90 \\
\hline 0.017 & 0 & 100 & 90 \\
\hline 0.018 & 0 & 100 & 90 \\
\hline 0.019 & 0 & 100 & 90 \\
\hline 0.02 & 0 & 100 & 90 \\
\hline
\end{tabular}

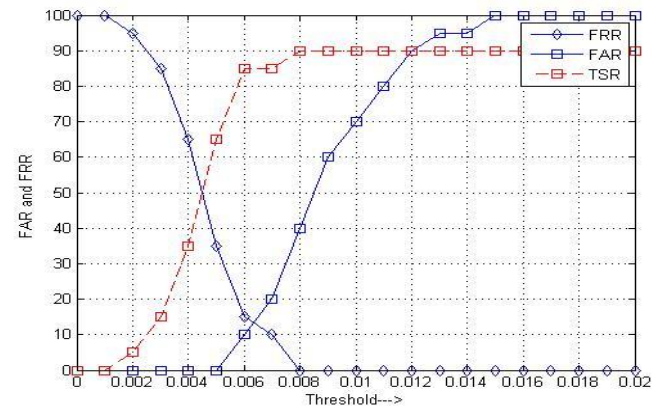

Fig.15: Plot of FRR FAR and TSR with threshold values for PID:POD of 20:20 for ORL database

5.2.5 Analysis using JAFFE face database: The variations of FRR, FAR, and TSR with threshold for PID of 5 and POD of 5 for JAFFE face database are given in table 10. The values of FAR and TSR increases as threshold values increases. The FRR values decreases with increase in threshold values. The maximum percentage TSR value is 100 and minimu m values of FRR and FAR are zeros as shown in the figure 16. The optimum values of TSR value is $100 \%$ with zero EER value

Table10: Variations of FRR, FAR, TSR with threshold for PID: POD of 5:5 for JAFFE

\begin{tabular}{|c|c|c|c|}
\hline Threshold & \%FRR & \%FAR & \% TSR \\
\hline 0 & 100 & 0 & 0 \\
\hline 0.001 & 100 & 0 & 0 \\
\hline 0.002 & 100 & 0 & 0 \\
\hline 0.003 & 60 & 0 & 40 \\
\hline 0.004 & 60 & 0 & 40 \\
\hline 0.005 & 20 & 0 & 80 \\
\hline 0.006 & 0 & 0 & 100 \\
\hline 0.007 & 0 & 0 & 100 \\
\hline 0.008 & 0 & 20 & 100 \\
\hline 0.009 & 0 & 40 & 100 \\
\hline 0.01 & 0 & 60 & 100 \\
\hline 0.011 & 0 & 60 & 100 \\
\hline 0.012 & 0 & 60 & 100 \\
\hline 0.013 & 0 & 80 & 100 \\
\hline 0.014 & 0 & 100 & 100 \\
\hline 0.015 & 0 & 100 & 100 \\
\hline 0.016 & 0 & 100 & 100 \\
\hline 0.017 & 0 & 100 & 100 \\
\hline 0.018 & 0 & 100 & 100 \\
\hline 0.019 & 0 & 100 & 100 \\
\hline 0.02 & 0 & 100 & 100 \\
\hline
\end{tabular}




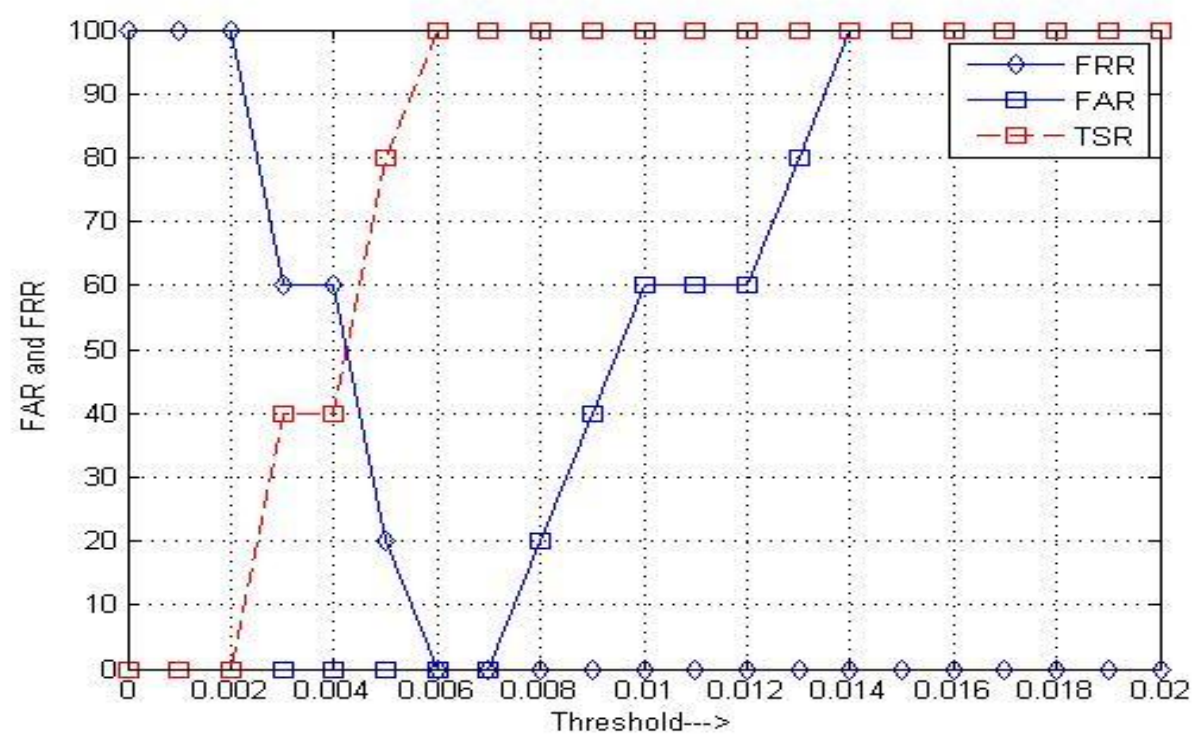

Fig. 16: Plot of FRR FAR and TSR with threshold values for PID:POD of 5:5 for JAFFE database

5.2.6 Analysis using Indian Males face database: The variations of FRR, FAR, and TSR with threshold for PID of 10 and POD of 10 for Indian Males face database are given in table 11. The values of FAR and TSR increases as threshold values increases. The FRR values decreases with increase in threshold values. The maximum percentage TSR value is $100 \%$ as shown in figure 17 . The optimum percentage TSR is 100 at EER value of zero.

Table 11: Variations of FRR, FAR, TSR with threshold for PID: POD of 10:10 for Indian Male

\begin{tabular}{|c|c|c|c|}
\hline Threshold & $\%$ FRR & $\%$ FAR & $\%$ TSR \\
\hline 0 & 100 & 0 & 0 \\
\hline 0.001 & 100 & 0 & 0 \\
\hline 0.002 & 90 & 0 & 10 \\
\hline 0.003 & 90 & 0 & 10 \\
\hline 0.004 & 30 & 0 & 70 \\
\hline 0.005 & 30 & 0 & 70 \\
\hline 0.006 & 20 & 0 & 80 \\
\hline 0.007 & 0 & 0 & 100 \\
\hline 0.008 & 0 & 50 & 100 \\
\hline 0.009 & 0 & 70 & 100 \\
\hline 0.01 & 0 & 80 & 100 \\
\hline 0.011 & 0 & 100 & 100 \\
\hline 0.012 & 0 & 100 & 100 \\
\hline 0.013 & 0 & 100 & 100 \\
\hline 0.014 & 0 & 100 & 100 \\
\hline 0.015 & 0 & 100 & 100 \\
\hline 0.016 & 0 & 100 & 100 \\
\hline 0.017 & 0 & 100 & 100 \\
\hline 0.018 & 0 & 100 & 100 \\
\hline 0.019 & 0 & 100 & 100 \\
\hline 0.02 & 0 & 100 & 100 \\
\hline
\end{tabular}




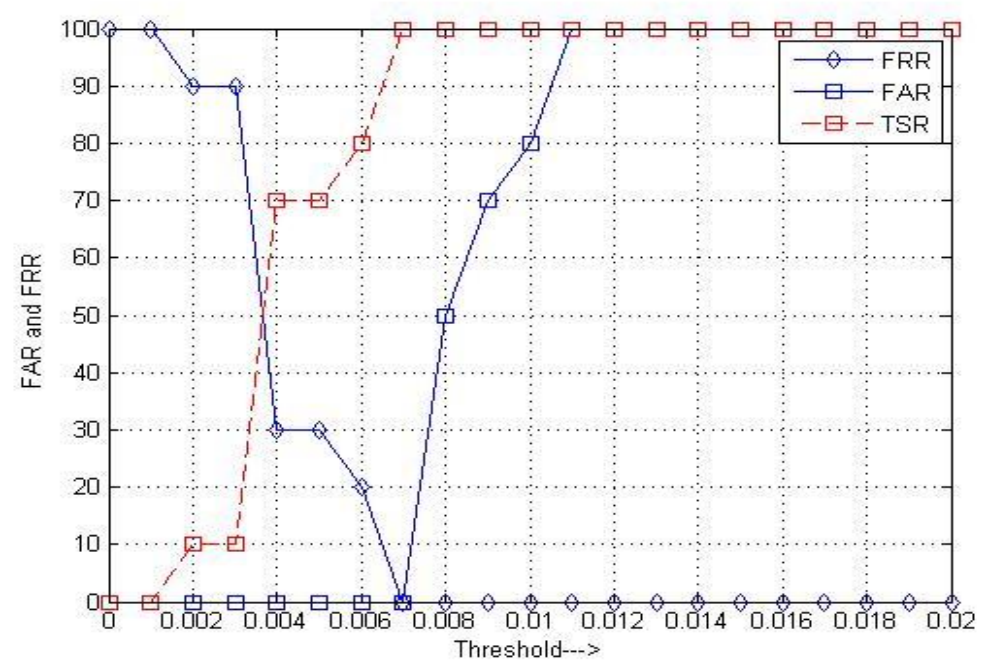

Fig. 17: Plot of FRR, FAR and TSR with threshold values for PID: POD of 10:10 for Indian Male database

5.2.7 Analysis using Indian-Females face database: The variations of FRR, FAR, and TSR with threshold for PID of 11 and POD of 11 for Indian-Female face database are given in table 12. The values of FAR and TSR increases as threshold values increases. The FRR values decreases with increase in threshold values. The maximum percentage TSR value is 100 as shown in figure 18 . The optimum TSR value is $90 \%$ at $10 \%$ EER value.

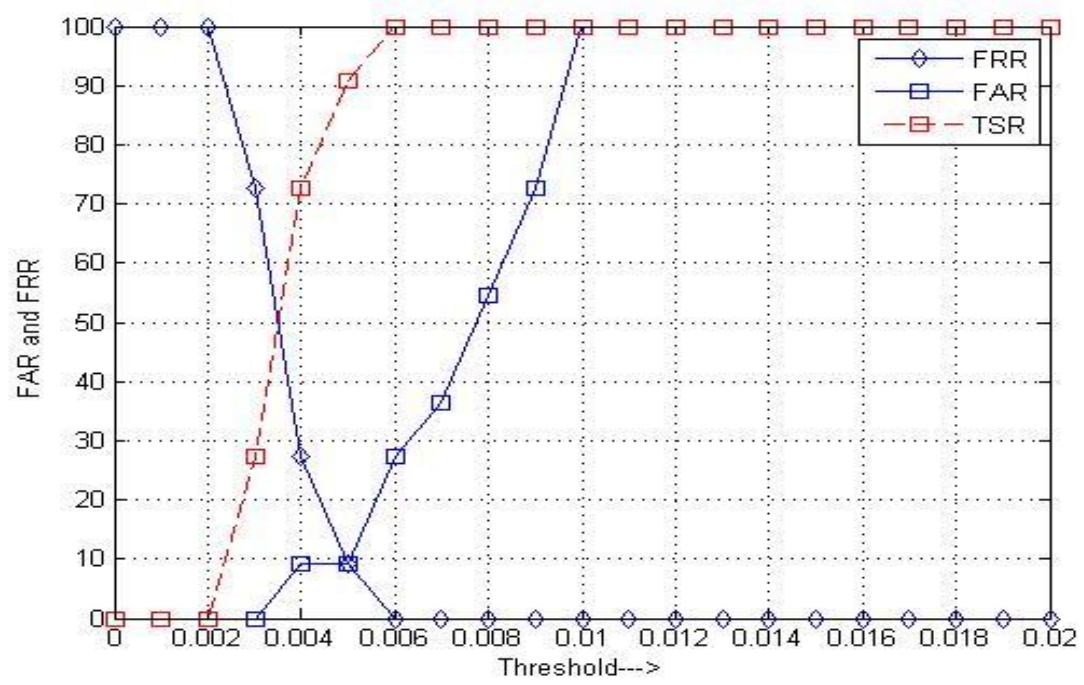

Fig.18: Plot of FRR FAR and TSR with threshold values for PID:POD of 11:11 for Indian - Female database

Table 12: Variations of FRR, FAR, TSR with threshold for PID:POD of 11:11 for Indian Females

\begin{tabular}{|c|c|c|c|}
\hline Threshold & \%FRR & \%FAR & \%TSR \\
\hline 0 & 1 & 0 & 0 \\
\hline 0.001 & 1 & 0 & 0 \\
\hline 0.002 & 1 & 0 & 0 \\
\hline 0.003 & 72.7272727 & 0 & 27.27272727 \\
\hline 0.004 & 27.2727273 & 9.0909091 & 72.72727273 \\
\hline 0.005 & 9.0909091 & 9.0909091 & 90.90909091 \\
\hline 0.006 & 0 & 27.2727273 & 100 \\
\hline 0.007 & 0 & 36.3636364 & 100 \\
\hline 0.008 & 0 & 54.5454545 & 100 \\
\hline
\end{tabular}




\begin{tabular}{|c|c|c|c|}
\hline 0.009 & 0 & 72.7272727 & 100 \\
\hline 0.01 & 0 & 1 & 100 \\
\hline 0.011 & 0 & 1 & 100 \\
\hline 0.012 & 0 & 1 & 100 \\
\hline 0.013 & 0 & 1 & 100 \\
\hline 0.014 & 0 & 1 & 100 \\
\hline 0.015 & 0 & 1 & 100 \\
\hline 0.016 & 0 & 1 & 100 \\
\hline 0.017 & 0 & 1 & 100 \\
\hline 0.018 & 0 & 1 & 100 \\
\hline 0.019 & 0 & 1 & 100 \\
\hline 0.02 & 0 & 1 & 100 \\
\hline
\end{tabular}

5.3 Variations of EER and optimum TSR for different face databases: The values of EER and TSR are computed for different face databses using DTCWT with various combinations of PID and POD are discussed in this section

5.3.1 Variations with $L$-space $K$ face database: The variations of EER and the corresponding percentage

Table 13: Variations of EER and optimu $m$ TSR for different values of PID and POD for L-Spacek

\begin{tabular}{|c|c|c|c|}
\hline PID & POD & \% EER & $\%$ opt. TSR \\
\hline 20 & 99 & 0.840336 & 99.159664 \\
\hline 40 & 79 & 1.257862 & 98.742138 \\
\hline 60 & 59 & 1.117318 & 98.882682 \\
\hline 80 & 39 & 1.680672 & 98.319328 \\
\hline 100 & 19 & 2.173913 & 97.826087 \\
\hline
\end{tabular}

5.3.2 Variations with Yale face database: The variations of EER and percentage TSR for different combinations of PID and POD for Yale database are tabulated in table 14 . The values of percentage optimum TSR is maximum is 100 and is almost constant around 90 for the variations of PID and POD.
TSR for different combinations of PID and POD for L-Spacek database are tabulated in table 13. It is observed that, the values of EER and percentage TSR are increased and decreased respectively with increase in PID. The values of percentage optimum TSR is maximum i.e., around 99 and is almost constant for the variations of PID and POD

Table 14: Variations of EER and TSR for different values of PID and POD for Yale

\begin{tabular}{|c|c|c|c|}
\hline PID & POD & $\%$ EER & $\%$ opt.TSR \\
\hline 4 & 11 & 0 & 100 \\
\hline 6 & 9 & 0 & 100 \\
\hline 8 & 7 & 6.666667 & 87.5 \\
\hline 10 & 5 & 6.666667 & 90 \\
\hline 12 & 3 & 6.666667 & 91.666667 \\
\hline
\end{tabular}

5.3.3 Variations with NIR face database: The variations of EER and percentage TSR for different combinations of PID and POD for NIR database are tabulated in table 15. The values of percentage optimum TSR is maximum i.e., 92.5 and is almost constant around 90 for the variations of PID and POD.

Table 15: Variations of EER and TSR for different values of PID and POD for NIR

\begin{tabular}{|c|c|c|c|}
\hline PID & POD & \% EER & \% opt. TSR \\
\hline 20 & 95 & 8.695652 & 90 \\
\hline 40 & 75 & 7.255814 & 92.5 \\
\hline 60 & 55 & 9.50495 & 90.247525 \\
\hline 80 & 35 & 11.502591 & 88.212435 \\
\hline 100 & 15 & 0.952381 & 93 \\
\hline
\end{tabular}

5.3.4 Variations with ORL face database: The variations of EER and percentage TSR for different combinations of PID and POD for ORL database are tabulated in table 16. The values of percentage optimum TSR is maximum i.e., 88 and is almost 
constant around 85 for the variations of PID and POD.

Table 16: Variations of EER and TSR for different values of PID and POD for ORL

\begin{tabular}{|c|c|c|c|}
\hline PID & POD & \%EER & \%opt.TSR \\
\hline 10 & 30 & 21.11 & 78.88 \\
\hline 20 & 20 & 13.33 & 85 \\
\hline 25 & 15 & 11.11 & 88 \\
\hline 30 & 10 & 12.72 & 86.96 \\
\hline 35 & 5 & 15 & 85 \\
\hline
\end{tabular}

5.3.5 Variations with JAFFE face database: The variations of EER and percentage TSR for different combinations of PID and POD for JAFFE database are tabulated in table 17 . The values of optimu m TSR is maximum i.e., 100 and is almost constant around 100 for the variations of PID and POD.

Table 17: Variations of EER and TSR for different values of PID and POD for JAFFE

\begin{tabular}{|c|c|c|c|}
\hline PID & POD & \% EER & \% opt. TSR \\
\hline 2 & 8 & 0 & 100 \\
\hline 4 & 6 & 0 & 100 \\
\hline 5 & 5 & 0 & 100 \\
\hline 6 & 4 & 10 & 90 \\
\hline 8 & 2 & 0 & 100 \\
\hline
\end{tabular}

5.3.6 Variations with Indian male face database: The variations of EER and percentage TSR for different combinations of PID and POD for Indianmale database are tabulated in table 18. It is observed that, the values of EER and percentage optimu m TSR are increased and decreased respectively with increase in PID. The maximum percentage optimum TSR is 100 .

Table 18: Variations of EER and TSR for different values of PID and POD for Indian-males

\begin{tabular}{|c|c|c|c|}
\hline PID & POD & \% EER & \% opt. TSR \\
\hline 4 & 16 & 15 & 85 \\
\hline 7 & 13 & 18.181818 & 81.818182 \\
\hline 10 & 10 & 0 & 100 \\
\hline 13 & 7 & 26.415094 & 73.584906 \\
\hline 16 & 4 & 30.555556 & 69.444444 \\
\hline
\end{tabular}

5.3.7 Variations with Indian female face database: The variations of EER and percentage TSR for different combinations of PID and POD for Indianfemales database are tabulated in table 19.

It is observed that, the values of EER and percentage TSR are increased and decreased respectively with increase in PID. The values of percentage optimum TSR is maximum i.e., 100 and is almost constant around 90 for the variations of PID and POD

Table 19: Variations of EER and TSR for different values of PID and POD for Indianfemales

\begin{tabular}{|c|c|c|c|}
\hline PID & POD & \% EER & \% opt. TSR \\
\hline 5 & 17 & 0 & 100 \\
\hline 8 & 14 & 8.695652 & 91.304348 \\
\hline 11 & 11 & 9.090909 & 90.909091 \\
\hline 14 & 8 & 13.888889 & 86.111111 \\
\hline 17 & 5 & 9.090909 & 85.026738 \\
\hline
\end{tabular}

5.4 Performance comparison of STWT and DTCWT for different face databases: The values of EER and optimum TSR for different databases are shown in table 20. It is observed that the values of EER are less in the case of DTCWT compared to STWT. The values of optimum TSR are high in DTCWT technique compared to STWT technique. The performance of face recognition using DTCWT is better compared to single wavelet transform for the reasons that (i) The DTCWT is shift invariant and (ii) The directional features are extracted.

Table 20: The variations of EER and TSR for different databases using STWT and DTCWT

\begin{tabular}{|c|c|c|c|c|c|c|}
\hline \multirow{2}{*}{$\begin{array}{c}\text { Data } \\
\text { base }\end{array}$} & \multirow{2}{*}{ PID } & \multirow{2}{*}{ POD } & \multicolumn{2}{|c|}{ STWT } & \multicolumn{2}{c|}{ DTCWT } \\
\cline { 4 - 7 } & & & $\%$ EER & $\begin{array}{c}\text { \% opt. } \\
\text { TSR }\end{array}$ & \%EER & $\begin{array}{c}\text { \% opt. } \\
\text { TSR }\end{array}$ \\
\hline L-Spacek & 60 & 59 & 8.45 & 90.12 & 1.117 & 98.883 \\
\hline Yale & 8 & 7 & 8.69565 & 87.5 & 6.6667 & 87.5 \\
\hline NIR & 60 & 55 & 12.7679 & 86.674 & 9.505 & 90.248 \\
\hline ORL & 20 & 20 & 16.6667 & 83.333 & 13.33 & 85 \\
\hline JAFFE & 5 & 5 & 10 & 90 & 0 & 100 \\
\hline Indian Males & 10 & 10 & 24 & 76 & 0 & 100 \\
\hline $\begin{array}{c}\text { Indian } \\
\text { Females }\end{array}$ & 11 & 11 & 14.091 & 85.91 & 9.091 & 90.91 \\
\hline
\end{tabular}




\section{CNCLUSIONS}

The face is a physiological Biometric trait used to recognize a person effectively. In this paper Face Recognition based on STWT and DTCWT using two dimensional Q-shift Filters proposed. The various available face databases such as L-space $\mathrm{k}$, ORL, YALE, JAFFE, NIR, Indian male, and Indian females are used to test the performance of the methods. The face images are resized in the pre-preprocessing unit. The features of face images are extracted using STWT and DTCWT. The features of database and Test images are compared using Euclidian distance.

\section{REFERENCES}

[1] Alaa Eleyan, Hasan Demirel and Hiiseyin Ozkaramanli,"Face Recognition using DualTree Wavelet Transform", IEEE International Symposium on Signal Processing and Information Technology, pp 7-11, 2008

[2] Zhongxi Sun, Wankou Yang1, Changyin Sun, Jifeng Shen, " Face Recognition Using DTCWT Feature-based 2DPCA", Chinese Conference on Pattern Recognition, pp 1-5, 2010

[3] R by Sun Yuehui and Du Minghui, "Robust Face Recognition for Illumination Removal Using DT-CWT and EMD" , IEEE International Conference on Communication Systems, pp. 357-361, 2008

[4] K.Jaya Priya and R.S Rajesh, "Local Statistical Features of Dual Tree Complex Wavelet Transform on Paralle logram Image Structure for Face Recognition with Single Sample", International Conference on Recent Trends in Information, Telecommunication and Computing, pp. 50-54, 2010

[5] Ravi J, Saleem S Tevaramani and K B Raja "Face Recognition using DT-CWT and LBP Features", International Conference on Computing, Communication and Applications, pp. 1-6, 2012

[6] N G Kingsbury, "A Dual-Tree Complex Wavelet Transform with Improved Orthogonality and Symmetry Properties", IEEE International conference on Image processing, vol.2, pp. 375-378, 2000

[7] Zeenathunisa, Jaya and Rabbani, "A Biometric Approach Towards Recognizing Face in Various Dark Illuminations," International Conference on Electronics Communication and Computing Technologies, pp 1-7, 2011.

[8] G Seyed Omid Shahdi and Abu-Bakar, "Frequency Domain Feature-Based Face Recognition Technique for Different Poses and Low-Resolution Conditions," International Conference on Imaging Systems and Techniques, pp 322-326, 2011.
[9] Ngoc-Son Vu and Alice Caplier, "Enhanced Patterns of Oriented Edge Magnitudes for Face Recognition and Image Matching," IEEE Transactions on Image Processing, vol. 21, no. 3, pp 1352-1365, 2012.

[10] Harin Sellahe wa and Sabah A. Jassim, "Image Quality based Adaptive Face Recognition," IEEE Transactions on Instrumentation and Measurement, vol.59, no.4, pp.805-813, April 2010.

[11] Ping-Han Lee, Szu-Wei Wu, and Yi-Ping Hung, "Illumination Compensation using Oriented Local Histogram Equalization and Its Application to Face Recognition," IEEE Transactions on Image Processing, vol.21, no.9, pp.4280-4289, September 2012.

[12] Vishal M.Patel, Tao Wu, Soma Biswas, P.Jonathon Phillips, and Rama Chellappa, "Dictionary-Based Face Recognition Under Variable Lighting and Pose," IEEE Transactions on Information Forensics and Security, vol.7, no.3, pp.954-965, June 2012.

[13] Abhijith Punnappurath and Ambasamudram Narayanan Rajagopalan "Face Recognition Across Non-Uniform Motion Blur, Illumination and Pose", IEEE Transactions on Image Processing, vol. 24, no. 7, pp. 20672082, July 2015

[14] Changxing Ding, Jonghyun Choi, Dacheng Tao and Larry S. Davis, "Multi-Directional Multi-Level Dual-Cross Patterns for Robust Face Recognition", IEEE Transactions on Pattern Analysis and Machine Intelligence, vol. 38, issue 3, pp. 518 - 531, July 2015.

[15] I.W. Selesnick, R.G. Baraniuk, and N.G.Kingsbury, "The Dual Tree Complex Wavelet Transform," IEEE Signal Processing Magazine, vol. 22, no.6, pp.123-151, Nov mber 2005

Authors' Profiles

H C Sateesh Kumar is a Associate Professor in the

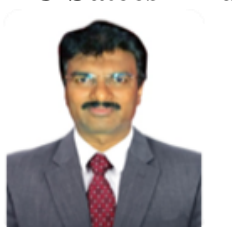
Dept of Electronics and Communication Engineering at Sai Vidya Institute of Technology, Bangalore. He obtained his B.E. degree in Electronics Engineering from Bangalore University. His specialization in Master degree was Bio-Medical Instrumentation from Mysore University and currently he is pursuing Ph.D. in the area of Image segmentation under the guidance of Dr. K B Raja, Professor, Dept of Electronics and Communication Engineering, University Visvesvaraya college of Engineering, Bangalore. He has over 21 research publications in refereed International Journals and Conference Proceedings. His area of interest is in the field of Signal Processing and Communication 
Engineering. He is the life member of Institution of Engineers (India), Institution of Electronics and Telecommunication Eng ineers and Indian society for Technical Education.

C Chowda reddy is Associate system Engineer at IBM india pvt. Ltd. He obtained Bachelors Degree in Electronics and Communication Engineering from SJCIT Chickballapur and M E degree in Electronics Engineering from University Visvesvaraya College of Engineering, Bangalore

Raja K.B. is a Professor, Department of Electronics

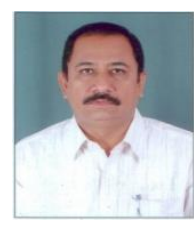
and Communication Engineering, University Visvesvaraya college of Engineering, Bangalore University, Bangalore. He obtained his B.E and M.E in Electronics and Communication Engineering from University Visvesvaraya College of Engineering, Bangalore. He was awarded Ph.D. in Computer Science and Engineering from Bangalore University. He has over 180 research publications in refereed International Journals and Conference Proceedings. His research interests include Image Processing, Biometrics, VLSI Signal Processing, computer networks.

Venug opal K.R. is currently the Principal and Dean,

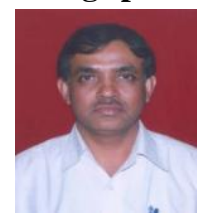

Faculty of Engineering, University Visvesvaraya College of Engineering, Bangalore University, Bangalore. He obtained his Bachelor of Engineering from University Visvesvaraya College of Engineering. He received his Master's degree in Computer Science and Automation from Indian Institute of Science, Bangalore. He was awarded Ph.D. in Economics from Bangalore University and Ph.D. in Computer Science from Indian Institute of Technology, Madras. He has a distinguished academic career and has degrees in Electronics, Economics, Law, Business Finance, Public Relations, Communications, Industrial Relations, Computer Science and Journalism. He has authored 27 books on Computer Science and Economics, which include Petrodollar and the World Economy, C Aptitude, Mastering C, Microprocessor Programming, Mastering $\mathrm{C}++$ etc. $\mathrm{He}$ has been serving as the Professor and Chairman, Department of Computer Science and Engineering, University Visvesvaraya College of Engineering, Bangalore University, Bangalore. During his three decades of service at UVCE he has over 520 research papers to his credit. His research interests include computer networks, parallel and distributed systems, digital signal processing and data mining. 